\title{
Zirconium Zr 89 DFO-Pertuzumab
}

National Cancer Institute

\section{Source}

National Cancer Institute. Zirconium Zr 89 DFO-Pertuzumab. NCI Thesaurus. Code C133822.

A radioimmunoconjug ate composed of pertuzumab, a humanized recombinant monoclonal antibody directed against the extracellular dimerization domain of the tyrosine kinase receptor human epidermal growth factor-2 (HER2; HER-2), and linked, via the chelator desferrioxamine (DFO), to the radioisotope zirconium Zr 89 (Zr89), with potential use in imaging of HER-2-expressing tumor cells upon positron emission tomog raphy (PET). Upon administration of zirconium Zr 89-DFO-pertuzumab, the pertuzumab moiety targets and binds to HER-2. After binding and internalization into HER-2-expressing tumor cells, Zr89 facilitates the visualization and detection of HER-2expressing tumor cells using PET. This assesses HER2-expression and may predict or evaluate the tumor's response to certain HER-2-targeting chemotherapeutics. 